\title{
Diffuse interstellar bands in the spectra of Herbig Ae/Be stars and related objects ${ }^{\star}$
}

\author{
G. A. Galazutdinov ${ }^{1,2,5}$, O. A. Galazutdinova ${ }^{2,5}$, and V. P. Grinin ${ }^{3,4}$ \\ 1 Center for Astronomy, Nicholas Copernicus University, Gagarina 11, 87-100 Toruń, Poland \\ e-mail: gala@astri.uni.torun.pl \\ 2 Special Astrophysical Observatory, Nizhnij Arkhyz 369167, Russia \\ e-mail: dolly@sao.ru \\ 3 Pulkovo Astronomical Observatory, St. Petersburgh 196140, Russia \\ e-mail: grinin@vG1723.spb.edu \\ ${ }^{4}$ Crimean Astrophysical Observatory, Crimea, 334413 Nauchny, Ukraine \\ e-mail: grinin@crao.crimea.ua \\ 5 Isaac Newton Institute of Chile, SAO Branch
}

Received 20 February 2003 / Accepted 17 April 2003

\begin{abstract}
We report a detection of weak diffuse interstellar bands (DIBs) in the close vicinity of 6 Herbig Ae/Be premain sequence stars. A common feature of these objects is the presence of a dusty shell where DIB carriers are apparently formed/destroyed. The possible influence of ultraviolet flux on carriers of diffuse interstellar bands is discussed as well as DIB to DIB intensity ratios in the spectra of the program stars and in the general interstellar medium.
\end{abstract}

Key words. stars: pre-main sequence - ISM: lines and bands

\section{Introduction}

The first report on Diffuse Interstellar Bands (DIBs) was published by Heger (1921), more than 80 years ago. At the present time, the number of known DIBs reaches 300 (Herbig 1975; Galazutdinov et al. 2000) and keeps growing with improving quality of the spectra: signal-to-noise ratio $(\mathrm{S} / \mathrm{N})$ reaching up to 1000 and resolving power $(R \equiv \lambda / \Delta \lambda)$, now exceeding 100000 .

In spite of the lack of any DIB carrier identification, some progress in understanding their origin cannot be denied. Different strength ratios of major DIBs along different lines of sight, clearly demonstrated by Krełowski \& Walker (1987), Krełowski \& Westerlund (1988) and Josafatsson \& Snow (1987), revealed a DIB origin in many carriers. Despite all conceivable forms of matter having already been proposed as possible carriers, from the negative hydrogen ion to dust grains, currently four items can be marked out: (i) dust grains, (ii) linear molecules based on a carbon skeleton, (iii) polycyclic aromatic hydrocarbons (PAHs), (iv) fullerenes. A search for appropriate carriers of diffuse interstellar bands is being

Send offprint requests to: G. A. Galazutdinov, e-mail: gala@astri.uni.torun.pl

* Based on data collected at the $2 \mathrm{~m}$ telescope operated on IC AMER Observatory (Terskol, Russia). conducted in close collaboration with laboratory researches. A final identification of a carrier is possible when a diffuse interstellar band profile coincides with that acquired in a laboratory (for more details see e.g. Herbig 1995; Fulara \& Krełowski 2000). Unfortunately, the observational determination of physical conditions inside interstellar clouds where DIBs originate is very difficult. Among the publications dealing with relations between diffuse interstellar bands and physical conditions of interstellar matter (see e.g., Cami et al. 1997; Snow et al. 2002) one should note the work of Krełowski et al. (1998), where it was firstly shown that the intensity of some DIBs depends on the ionization level of the obscuring matter.

The above indicates that it may be useful to observe DIBs formed in circumstellar shells, where a reconstruction of physical conditions seems to be much more reliable. The first and accidental observational evidence of short-time-scale DIB variability (Galazutdinov et al. 1999) demonstrated the possibility of synthesis/destruction of DIB carriers in the close vicinities of stars. However, the observations concern a variable object, characterized by irregularity. Further observations did not show any evident variability. We thus found it interesting to study diffuse interstellar bands in stars still related to some remnants of their parent clouds - namely, Herbig Ae/Be stars. Discovered about 40 years ago (Herbig 1960), these objects are pre-mainsequence, intermediate-mass $\left(2-10 M_{\odot}\right)$ stars, characterized 
by variable brightness, polarization and emission lines, which implies a significant amount of circumstellar matter around any of them. There are reports on detecting diffuse interstellar bands in these objects, see e.g. Miroshnichenko et al. (2001). Short-time-scale (of days or even hours) variability in extrastellar lines (e.g. Na I), accompanied by DIBs ${ }^{1}$ (Natta et al. 2000; Vieira et al. 1999) has also been reported. Thus, our idea may be described as "a search for DIB variability in the spectra of Ae/Be stars acquired in different phases of variability". Possible variations of other spectral features, formed in the circumstellar shell, may help to reconstruct the physical conditions that facilitate the formation or preservation of the DIB carriers. We report here the first step of this project - observations of 6 Herbig Ae/Be stars (Thé et al. 1994), which are rather bright objects, shadowed by surrounding shells with a relatively low abundance of obscuring circumstellar matter.

$\mathrm{Ae} / \mathrm{Be}$ Herbig stars do not form a homogeneous class of objects but all of them are characterized by strongly variable spectra induced by different mechanisms active in relatively dense (as compared to the general interstellar medium) matter in the close vicinity of any star. There are difficulties with interpreting such a complex pattern of observed variabilities including extended chromosphere, stellar wind, circumstellar shells and the impact of a surface magnetic field. However, despite these difficulties, the objects offer possibilities to estimate the physical conditions affecting the formation of DIB carriers: at least due to the possibility of comparing models and observed variability of extra-stellar spectral features.

Indeed, in a number of studies dedicated to short time variability detected in the close vicinity of Ae/Be stars, the authors did not pay attention to diffuse interstellar bands. A few mentions of the presence of diffuse interstellar bands in the spectra of HerbigAe/Be stars (i.e. Miroshnichenko et al. 2001) have not brought any additional information concerning DIBs except their visibility. In the recent work of Rodgers et al. (2002) the authors published measurements of the DIB 6283 in the spectrum of the variable star RR Tau, possibly revealing some variability (see Table 1 in their paper). They noted, however that "A narrow feature at $6283 \AA$ seen in all spectra is likely due to telluric absorption" (p. 408). The reason is that the moderate resolution of their spectra does not make it possible to completely remove the telluric lines from the profile of DIB 6283.

We therefore consider our article as a first attempt to study the behavior of diffuse interstellar bands seen in the spectra of Herbig Ae/Be stars.

\section{The observational data}

The spectra have been collected using a Coudé échelle spectrometer (Musaev et al. 1999) mounted on the 2-m telescope of the IC AMER Observatory at Peak Terskol in the Northern Caucasus. The spectrometer forms the spectrum on a Wright Instruments CCD $1242 \times 1152$ matrix (pixel size $22.5 \mu \mathrm{m} \times$ $22.5 \mu \mathrm{m}$ ) camera covering the range from 3500 to $10100 \AA$

\footnotetext{
${ }^{1}$ If a spectrum shows DIBs at all, then sodium D lines are always present and strong.
}

Table 1. The list of observed stars and measurement data. Column headings: HD - HD number; SpL - spectral and luminosity class; $V$ - visual apparent magnitude; DIB 5780 and DIB 5797 - equivalent

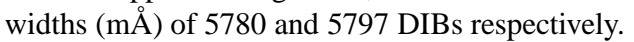

\begin{tabular}{rlrrr}
\hline \hline $\mathrm{HD}$ & SpL & DIB 5780 & DIB 5797 \\
\hline 31648 & A5 Vp $^{a}$ & 7.73 & $59 \pm 5$ & $<5$ \\
36917 & A0V $^{c}$ & 8.03 & $83 \pm 7$ & $52 \pm 5$ \\
50138 & B5 III $^{b}$ & 6.583 & $51 \pm 6$ & $19 \pm 4$ \\
179218 & A0 IVe $^{a}$ & 7.2 & $107 \pm 6$ & $<11$ \\
190073 & A2 IVev $^{a}$ & 7.2 & $85 \pm 5$ & $<12$ \\
250550 & A0 $^{c}$ & 9.57 & $107 \pm 6$ & $11.7 \pm 2$ \\
\hline
\end{tabular}

${ }^{a}$ Mora et al. (2001); ${ }^{b}$ Jaschek et al. (1992); ${ }^{c}$ The SIMBAD database (http://simbad.u-strasbg.fr).

with $R=45000$ (2.5 pixels) in a single exposure. For $\lambda<$ $7000 \AA$ spectra are recorded without any gaps between orders.

The spectra were reduced using the DECH code (Galazutdinov 1992) which provides all standard stages of CCD image and spectra processing.

For wavelength calibration, we used the scattered daylight spectrum which provided at least 15-20 points in each spectral order. The laboratory wavelengths of the solar spectrum lines are taken from the tables of Pierce \& Breckinridge (1973). When removing the telluric lines, we used the spectra of rapidly rotating non-reddened B stars (HD 120315, HD 116658, HD 218045).

General properties of the observed stars are listed in Table 1 together with the results of equivalent width measurements of 5797 and $5780 \AA$ DIBs. Measurements are done using the direct integration method or gauss de-blending in case of stellar contamination.

Heliocentric radial velocities of the "interstellar" features, seen in the program stars, are given in Table 2. Measurements are done by manually matching the investigated profiles with their mirror images (spectrum fragment with opposite wavelength direction). This method makes it possible to measure radial velocities confidently in cases where profiles are characterized by complexity of shape and/or if they are very broad. We estimate the precision of our measurements to be slightly better than $1 \mathrm{~km} \mathrm{~s}^{-1}$; this mostly depend on spectral resolution and spectral-to-noise ratio.

However, Table 2 show some difference between the radial velocities of DIBs and those of atomic IS lines. However, this is due to a strong asymmetry of the DIB, which commonly varies from star to star. The adopted wavelength of the DIB is determined from the high-quality spectrum of HD 23180 (Galazutdinov et al. 2000) where, e.g. DIB 5780 is relatively symmetric. Generally speaking, the task of radial velocity measurements of broad and non-symmetrical features is not trivial and requires making a preliminary (and subjective) decision: where to measure it - in the core, at full width at half maximum or somewhere else? The example with DIB 5780 illustrates again the importance of homogeneous investigations of radial velocities of DIBs and the necessity of correcting for the differences in the methods used in the various compilations. 
Table 2. Radial velocity measurements data $\left(\mathrm{km} \mathrm{s}^{-1}\right)$. Column headings: star - HD number; $l, b$ - Galactic longitude and latitude; $K \mathrm{I}-K \mathrm{I}$ line at $7699 \AA$ A $D 1, D 2$ - Na I lines; $H, K$ - Ca II lines; 5780, 5797 - diffuse interstellar bands at 5780 and $5797 \AA$ respectively.

\begin{tabular}{lrrrrrrrrr}
\hline \hline Star & $l$ & $b$ & $K$ I & $D 1$ & $D 2$ & $H$ & $K$ & 5780 & 5797 \\
\hline HD 31648 & 173.47 & -7.9 & 14.7 & 15.4 & 16.2 & 15.7 & 14.5 & 14 & 16 \\
HD 36917 & 209.12 & -19.57 & 27.1 & 27.5 & 27.6 & 29.5 & 26.5 & 76 & 32 \\
HD 50138 & 219.15 & -3.14 & 40.5 & 40.7 & 40.9 & 35.6 & & & \\
& & & 19.3 & 19.6 & 20.5 & 23.1 & 24.5 & 24 & 37 \\
HD 179218 & 49.21 & 2.89 & & -17.1 & -15.6 & -13.9 & -15.8 & -8 & -22 \\
HD 190073 & 46.5 & -13.1 & & 0.3 & 1 & 2.5 & 1 & -8 & -2 \\
& & & & -17.4 & -16.1 & -15 & -20 & & \\
HD 250550 & 192.62 & -3.04 & & 15.7 & 17.2 & 15.5 & 15.2 & 11 & 10.5 \\
\hline
\end{tabular}

\section{Observed stars}

Circumstellar dust disks are most easily studied while they are still young and are best seen around the brightest pre-mainsequence stars - Herbig Ae/Be type. However, there is a significant difference between Ae and Be classes: while Herbig Ae type stars are surrounded by disks, as shown by, e.g. detection of their millimeter flux, Herbig Be stars, in general, are not (Natta et al. 2000). We observed and detected diffuse interstellar bands in both kinds of objects as reported below.

\subsection{HD 31648}

The star is a pre-main sequence object with surrounding debris disk (Sitko et al. 1999). The star is situated $131 \pm 20 \mathrm{pc}$ from the Sun and possesses a silicate emission feature at $10 \mu \mathrm{m}$ also observed in solar system comets. This suggests that the material in this system has a processing history similar to that of dust in the solar system prior to the time when grains were incorporated into comets.

The spectral features characterizing this star are shown in Fig. 1. There is clear emission in the K I (7699 $\AA$ ) and Na I (D1) lines that are characteristic features of Herbig $\mathrm{Ae} / \mathrm{Be}$ stars, lying in regions of star formation. Such regions include "obvious" obscuration due to dust, and illuminate nearby reflection nebulae (Herbig 1960). Mannings \& Sargent (1997) and Mannings et al. (1997) have mapped the thermal dust millimeter-continuum and gaseous CO emission in HD 31648, where the surrounding dust disk has an extent of $85 \mathrm{AU}$ (HWHM). The disk is massive and may provide star brightness glimpses (Sitko et al. 1999). Concerning the disk size, it is interesting to cite the recent article by Hinz et al. (2001) - they estimated disk sizes using nulling interferometry and suggested that the extent of mid-IR emission around Herbig Ae stars may be much smaller than current models predict. They constrain $\sim 90$ percent of the $10 \mu \mathrm{m}$ flux to be within an approximately $20 \mathrm{AU}$ diameter region.

Concerning our observational data, we would like to draw attention to very weak narrow DIBs, seen in the spectrum of HD 31648 (Fig. 1). We show two of them - DIB 5797 and DIB 5780. The latter is very weak - this fact may be considered a demonstration of the relatively high ionization rate in the region where the carriers of such DIBs are formed. As will be shown below, weakness of narrow DIBs is a common feature of $\mathrm{Ae} / \mathrm{Be}$ stars, at least in the currently observed sample.

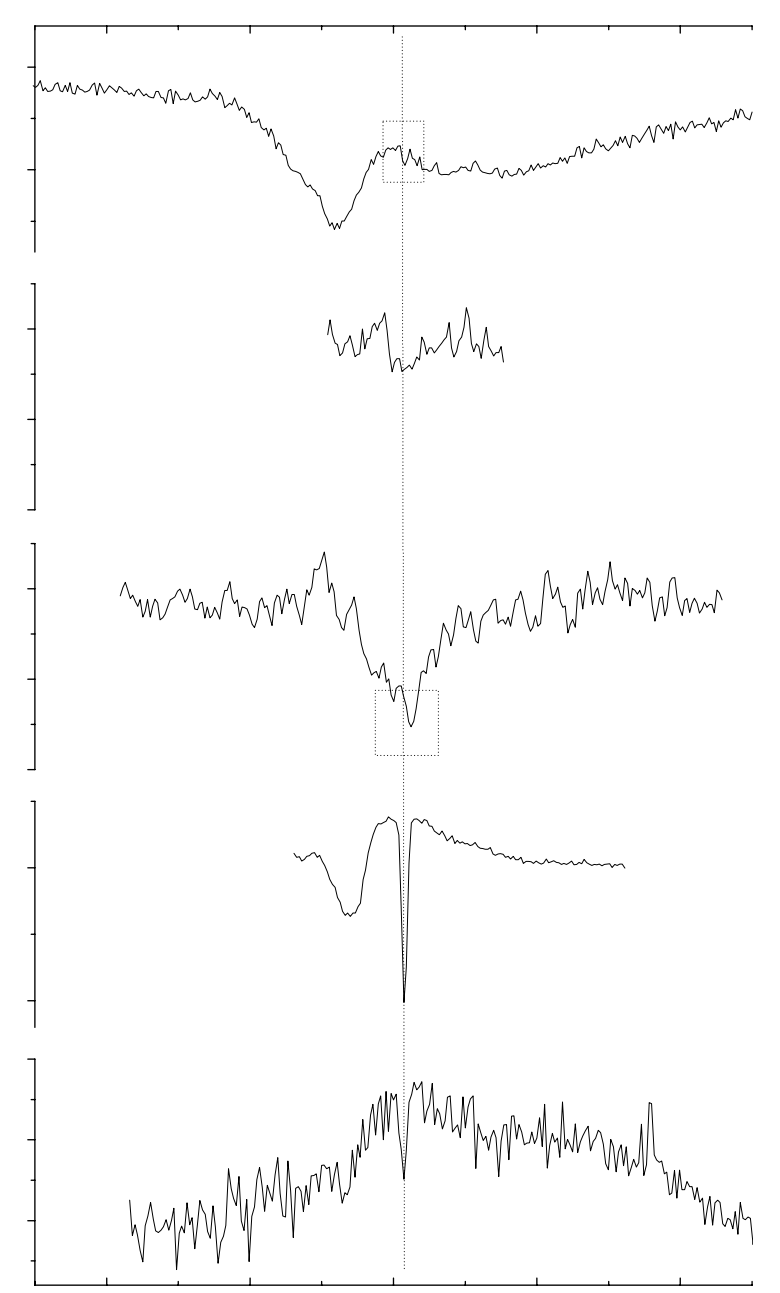

Fig. 1. Spectral features in the "late" Ae/Be Herbig star HD 31648. Note the sharp feature in the center of DIB 5780 (it is a stellar Fe II line) and the extremely weak $\mathrm{Ca}$ II $\mathrm{H}$ line.

\subsection{HD 36917}

The star is a member of the young Orion nebula cluster (age $\sim 1 \mathrm{Myr}$ ) and is known to have a gas-dust shell and/or shelldisk (Hovhannessian \& Hovhannessian 2001). A recent study of HD 36917 by Manoj et al. (2002) reported the absence of typical Herbig Ae/Be emission features in the near infra-red. This suggests the absence of hot dust in the vicinity of the 


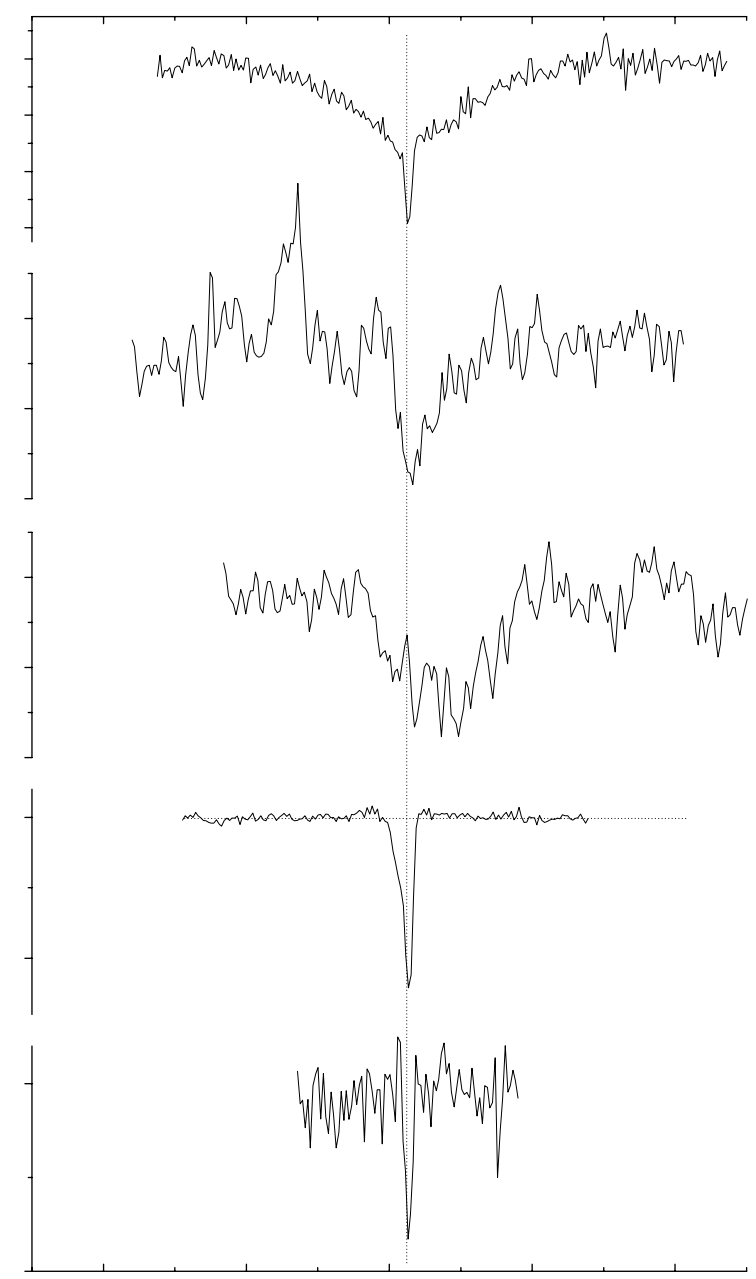

Fig. 2. Features seen in the spectrum of HD 36917. Note the weak emission in Na I (D2). Red-shifting is a common characteristic of DIBs, seen in the spectra of the Orion nebula stars.

central star. However, the far-infrared emission excess discovered here (Hovhannessian \& Hovhannessian 2001), is evidently due to both the thermal emission of grains and to the emission originating in free-free transitions of electrons in the gas-dust shell. Our observations in the 3500-10000 $\AA$ wavelength region discovered that the HD 36917 spectrum contains weak emission, seen only in the $\mathrm{Na}$ I (D2) line.

Manoj et al. (2002) found the star to be a young Vega-like object because "the fractional infrared luminosities $\left(L_{\mathrm{IR}} / L_{*}\right)$ and the dust masses computed from IRAS fluxes are systematically lower than those found for Herbig Ae/Be stars but higher than those for Vega-like stars". They may be right: the star shows the weakest emission feature in our sample. Anyway, the relatively extended disk around HD 36917 is likely the place where the carriers of observed DIBs are situated. The narrow $5797 \AA$ feature is here relatively deep (Fig. 2).

\subsection{HD 50138}

A combined study of the spectral and photometric characteristics of the star led Jaschek et al. (1992) to estimate the spectral

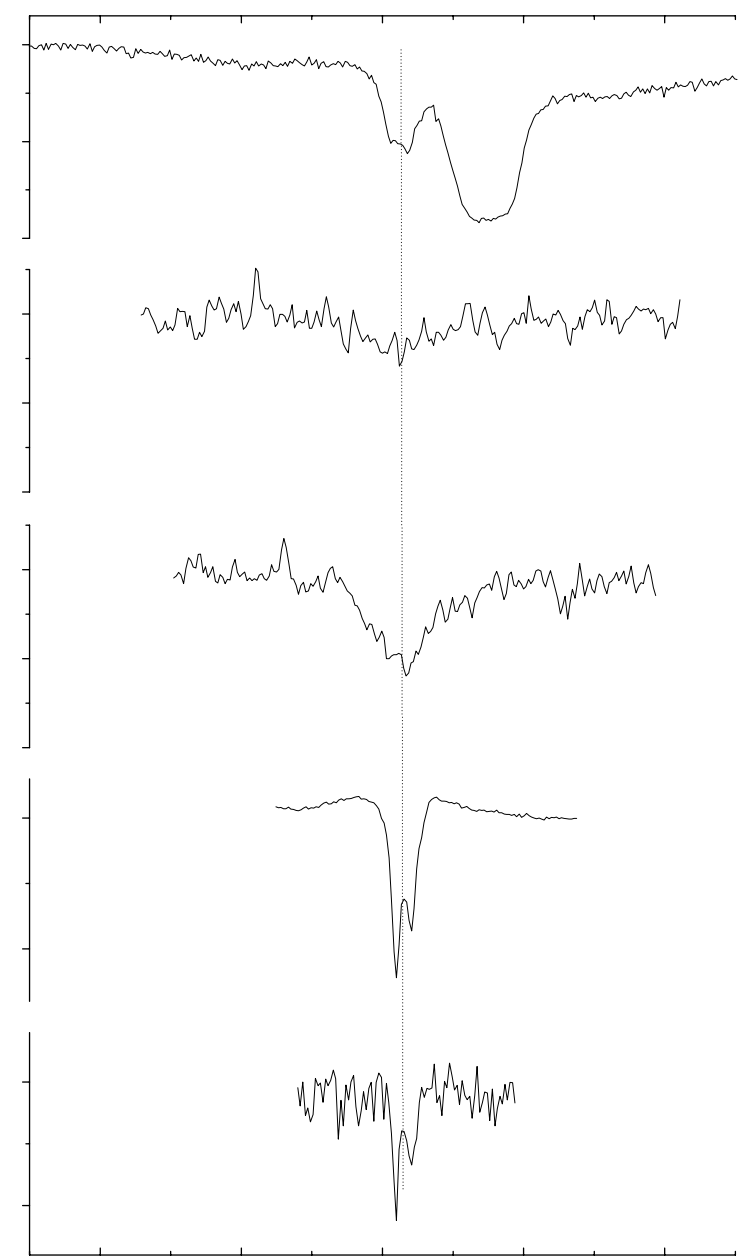

Fig. 3. Spectral features seen in the spectrum of HD 50138.

type of HD 50138 as B5 III. The star lies at a distance of 290 pc $(+90,-50 \mathrm{pc})$ and possibly is a pre-main-sequence Herbig Be star because of the observed asymmetries in the red wings of the UV stellar lines of C IV and Fe III (Grady et al. 1994) and the variability in optical lines, interpreted as due to accretion of the disk matter onto the star (Pogodin 1997). The latter author also interpreted the rapid variability of $\mathrm{H}_{\alpha}$ and $\mathrm{NaI} D$ lines as evidence of discrete structure within the stellar wind.

HD 50138 is also suspected to be a binary (Cidale et al. 2001). However, the possible binarity of the star still needs to be confirmed spectroscopically. On the basis of ultraviolet spectropolarimetry Bjorkman et al. (1998) found strong evidence of a thin gaseous disk around the star with a small inclination of the disk away from edge-on with respect to the line of sight. They suggested that the envelope of HD 50138 probably has a rather large density contrast between the equatorial and polar regions.

There is a problem of classification of HD 50138. It has usually been put either in the group of B[e] stars (Bopp 1993) or in the group of Herbig Ae/Be stars (Morrison \& Beaver 1995). But this is not important for us as the star is well-known to be a variable, surrounded by absorbing matter which causes the observed diffuse bands (Fig. 3). 


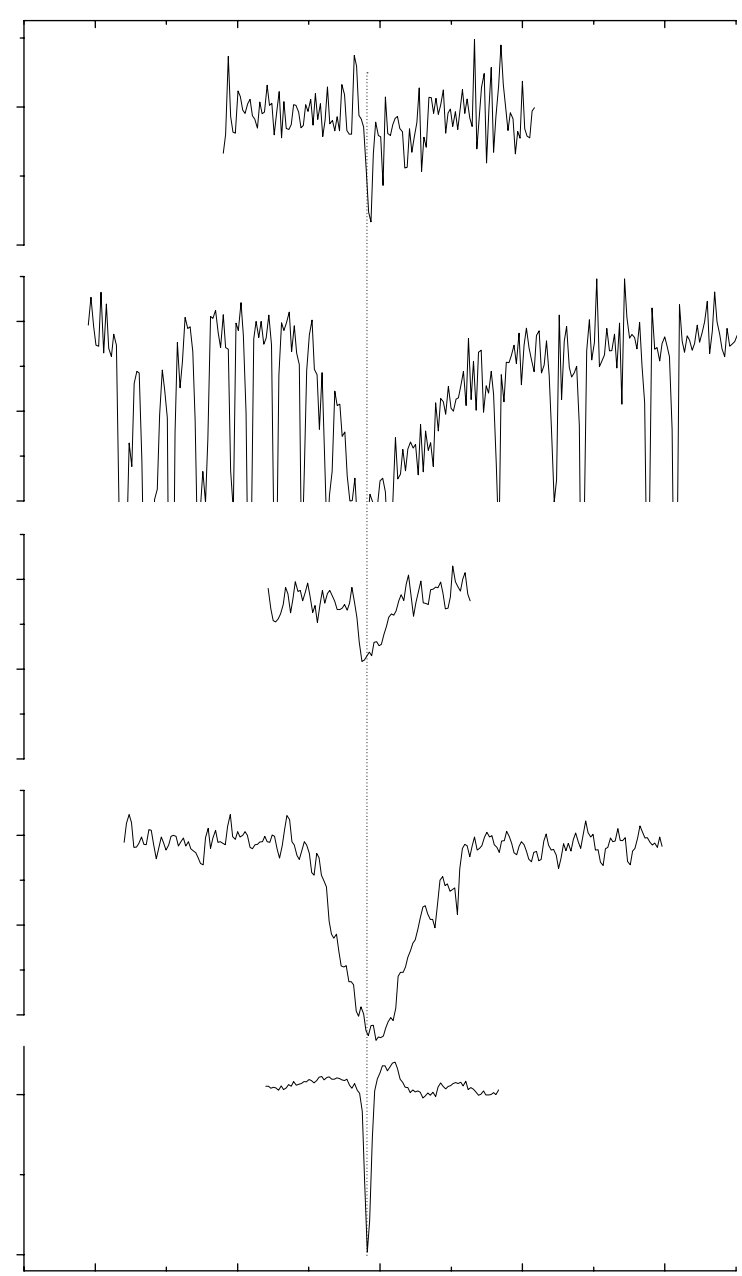

Fig. 4. Features seen in the spectrum of HD 179218. The DIB 6284 profiles contain sharp remnants of unremoved telluric lines.

\section{4. $H D 179218$}

HD 179218 exhibits a large near- and far-infrared excess: it shows infrared emission from crystalline silicates (Harker et al. 2002). Remarkable variations are observed in its $\mathrm{H}_{\alpha}$ profile (Miroshnichenko et al. 1999). That phenomenon was also observed in some classical Be and Herbig Ae/Be stars. This star does not belong to any known star-formation region, but it shows a compact nebula which is similar to those of isolated young intermediate-mass stars. It is probably a pre-mainsequence Herbig Be star (Miroshnichenko et al. 1999).

In Fig. 4 we show the features seen in its spectrum. Note the emission in the NaI $D 1$ line. We show both the 5780 and $6284 \AA$ broad diffuse interstellar bands, which are wellcorrelated (Moutou et al. 1999). Remnants of the telluric lines seen in the profile of DIB 6284 are due to an improper spectrum of the divisor star, observed with much lower air-mass. A weak, "narrow" DIB at $5797 \AA$ may reflect a relatively high ionization level in the vicinity of HD 179218 as in HD 31648.

\section{5. $H D 190073$}

HD 190073 is an early type (A2III - Divan \& Zorec 1982) emission line star with a significant IR excess. The star is well known for its double Ca II violet displaced shell absorption components which are variable in time (Struve \& Swings 1942; Surdej \& Swings 1976). It is a giant with a significant magnetic field (Babcock 1958) and displays a strong wind (expanding chromosphere). As is shown in Fig. 5, shell lines of single ionized metals display large expansion velocities in contrast to $\mathrm{Na}$ I and $\mathrm{K}$ I faint emission yielding low expansion velocities. Surdej \& Swings (1977) considered these rapid and sudden variations in the extended atmosphere of HD 190073 in terms of the ejection of $\mathrm{Ca}$ II ions from the outer atmosphere by radiative forces, acting selectively via a resonance scattering mechanism.

Figure 5 demonstrates some spectral features seen in our spectrum of the star. We made a point of showing both Ca II lines $(H$ and $K)$ to emphasize the complex structure seen in lines of that ion only. It is interesting that these components at $\sim-200$ and $\sim-300 \mathrm{~km} \mathrm{~s}^{-1}$ have been observed during more than half a century! This means that they cannot be explained in terms of sudden mass ejection as in, e.g. novae. The star represents the only case where we do not see absorption in the $\mathrm{K}_{\mathrm{I}}$ (7699 ̊) line but DIB 5797 exists anyway. We will postpone interpreting this phenomenon until we have a set of spectra big enough to detect and analyze radial velocity shifts with time.

\section{6. $H D 250550$}

The Herbig Ae star HD 250550 lies at a distance of 700 pc and possesses a strong stellar wind and a dense chromosphere (Cohen 1973). Satellite observations (FUSE) discovered several absorption features from molecular hydrogen and showed that $\mathrm{H}_{2}$ is thermalized up to $J=3$ and its radial velocity is identical to that of the surrounding molecular cloud's velocity (Martin et al. 2002). The detection of a number of absorption lines arising from excited levels of atomic species like $\mathrm{N}$ I, Cl I, Cl II, P II, Fe II, and Fe III reveals a complex circumstellar environment. This may suggest that quite a large part of the original molecular cloud that collapsed to form the star is still present in the vicinity of the star.

Catala et al. (1991) considered short-time (hours!) variability, seen in the Ca II K line. They attributed the narrow absorption in the center of the line to a circumstellar shell. That line is also seen in our Fig. 6. Let us emphasize that Catala et al. (1991) reported several periods of variability, attributed to different components of the Ca II K profile. They proposed that the periodic part of these variations should be considered in terms of magnetic stream model, where the star surface magnetic field controls the structure of fast and slow streams, alternating on the line of sight.

\section{Discussion}

Diffuse interstellar bands are detected in the spectra of 6 objects observed by us. These objects belong to different spectral classes: Ae as well as Be, but all are relatively hot - no one is 


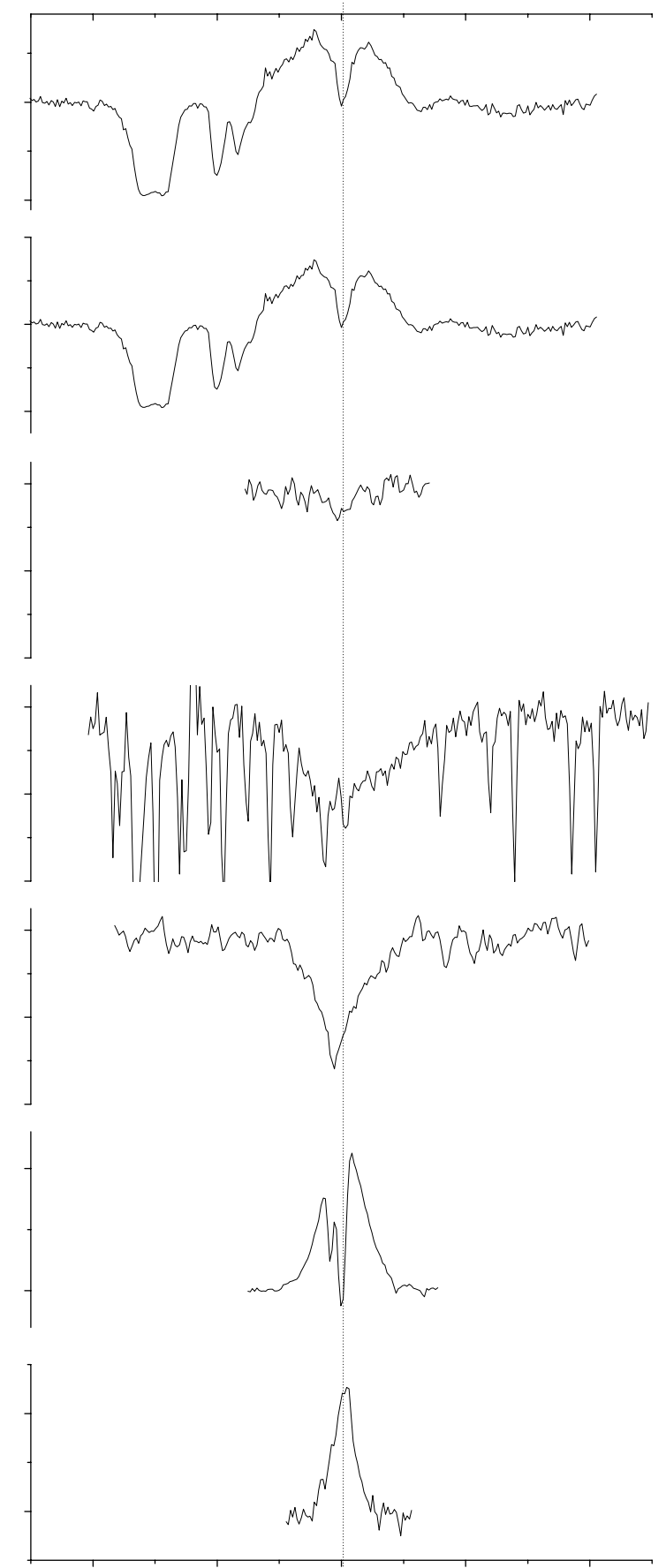

Fig. 5. Complex spectral features of HD 190073. The central part of DIB 5780 is blended with the Fe II line. The blue shifted double components seen in the Ca II lines were already reported by Merrill (1950). DIB 6284 is contaminated by sharp telluric lines.

cooler than A3. It is no accident that separating pure DIB profiles from later type stellar spectra - rich in atmospheric lines, is a very difficult task. Observations of objects such as earlytype Ae and Be stars prove that profiles of diffuse interstellar bands are mostly free of blending with stellar lines.

Our measurements of the equivalent widths of DIBs at 5797 and $5780 \AA$ (see Table 1) demonstrate that in most cases the intensity of DIB 5797 is much lower than that of DIB 5780

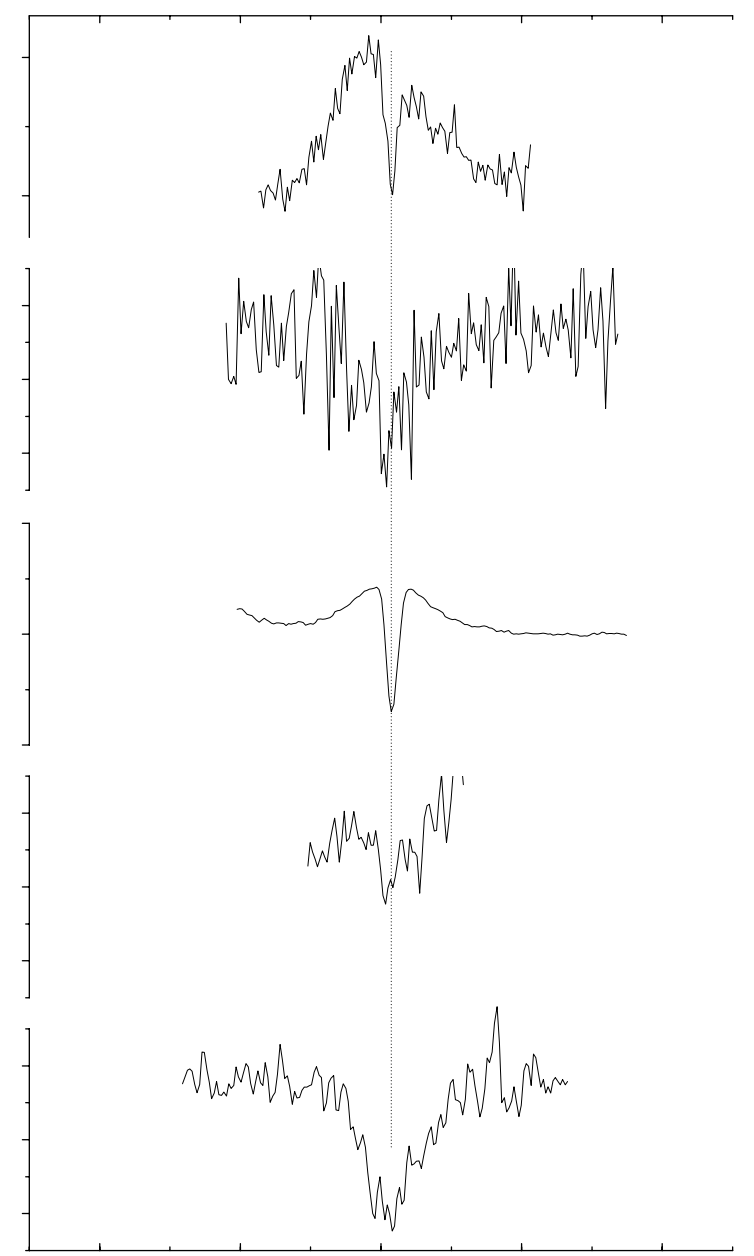

Fig. 6. Broad emission is seen in the atomic lines of HD 250550. Note very weak "narrow" DIBs (6614 and 5797) in contrast to the clear DIB 5780.

with one exception (HD 36917, which, seemingly is a Vegalike star). We think this is not accidental but caused by physical conditions. The idea is as follows: the broad DIBs like 5780 or $6284 \AA$ are seen if any DIBs are observable at all. These DIBs are observable even in Ori OB1 association stars (HD 37022, HD 37023, HD 34076 etc.) where the UV flux, originating in several very hot, young stars, is very strong. For these stars, they (DIBs) are, however, very weak, in relation to the observed $E(B-V)$. Apparently the carriers of such DIBs are very stable against dissociation by energetic UV-photons. Most probably this is why we see 5780-like DIBs in the close vicinity of Herbig Ae/Be stars, where the proximity of a central star compensates its low UV irradiation flux. Apparently the DIB 5797 carrier is much less likely to survive in an intense UV-irradiation (Krełowski et al. 1998) than the DIB 5780 carrier. On the other hand, $\mathrm{B}[\mathrm{e}]$ stars are surrounded by circumstellar disks. The intensity of DIBs is unusually weak in their spectra in spite of a sufficient $E(B-V)$ value: e.g. HD 164284 (Galazutdinov et al. 1997). Perhaps the carriers are destroyed in the close vicinity of hot stars and preserved in late B and early A stars. 


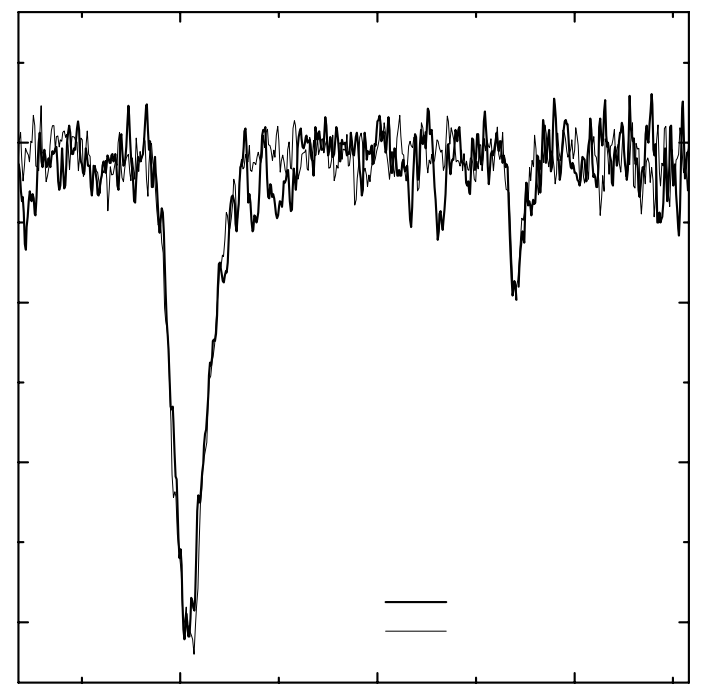

Fig. 7. The comparison of the general average spectrum of all the considered stars (except HD 36917) with a spectrum of a well-known $\sigma$ type object: HD 144217. In both spectra the 5797 feature is normalized to unity.

Finally, we constructed the general average of the observed target spectra (Table 1) in the region of the DIBs 5780 and $5797 \AA$ and compared it with the spectrum of a typical $\sigma^{2}$ type object: HD 144217. For clarity both spectra are normalized to match the DIB 5797 depth (Fig. 7). The normalization made the DIBs identical, which indicates that the already introduced $\sigma$-type cloud is simply a heavily irradiated object. This is consistent with the relatively low intensity (considering the value of $E(B-V))$ of e.g. the $\mathrm{K}$ I interstellar line compared to what is found in $\zeta$-type objects.

As far as one can judge from our spectra, DIBs observed towards the targets of Table 1 seemingly originate in the same medium (presumably circumstellar shells) as gaseous lines (K I or $\mathrm{NaI}$ ). It cannot be excluded that the sightlines towards our objects intersect additional interstellar clouds, but they would be very unlikely to move with exactly the same radial velocities.

The lack of emission wings in DIBs originating in circumstellar shells (similar to those observed in $\mathrm{Na}$ I and/or K I lines) gives another support to the hypothesis connecting them to either large molecules or dust grains. In such a carrier any absorbed photon is quickly distributed among internal degrees of freedom which prevents its simple re-emission.

The best confirmation of the proposed idea concerning the possibility of synthesis/destruction of DIBs carriers in circumstellar shells of $\mathrm{Ae} / \mathrm{Be}$ stars, would be a detection of

\footnotetext{
${ }^{2}$ Krełowski \& Walker (1987) proposed the division of diffuse clouds in two types based on the intensity ratio of the strong diffuse bands at 5780 and $5797 \AA$. The $\sigma$-type clouds (after the prototype star $\sigma \mathrm{Sco}$ ) is characterized by low intensity of interstellar molecules and "narrow" DIBs like $5797 \AA$ A. The $\zeta$ type clouds (after the prototype star $\zeta$ Oph) show strong molecular lines and deep diffuse bands like $5797 \AA$.
}

short-time-scale DIB variability in agreement with that in other, well-identified features.

Acknowledgements. The authors want to express their thanks to the Russian Foundation for Basic Research for financial support under the grant No 02-02-174423 and the Russian Federal program "Astronomy". The project was financially supported by the Polish Committee for Scientific Research $(\mathrm{KBN})$ under the grant 2 P03D 01923.

\section{References}

Babcock, H. W. 1958, ApJS, 3, 141

Bjorkman, K. S., Miroshnichenko, A. S., Bjorkman, J. E., et al. 1998, ApJ, 509, 904

Bopp, B. W. 1983, IBVS, 3834, 1

Catala, C., Czarny, J., Felenbok, P., Talavera, A., \& Thé, P. S. 1991, A\&A, 244, 166

Cidale, L., Zorec, J., \& Tringaniello, L. 2001, A\&A, 368, 160

Cohen, M. 1973, MNRAS, 164, 395

Divan, J., \& Zorec, J. 1982, Proc. International Coll. The Scientific Aspects of the Hipparcos Missions, ESA SP-177

Fulara, J., Jakobi, M., \& Maier, J. P. 1993, Chem. Phys. Lett., 211, 227

Fulara, J., \& Krełowski, J. 2000, New Astron. Rev., 44, 581

Galazutdinov, G. A. 1992, Prep. Spets. Astrof. Obs., No. 92

Galazutdinov, G. A., Krełowski, J., Musaev, F. A., \& Bikmaev, I. F. 1997, MNRAS, 292, 414

Galazutdinov, G. A., Krełowski, J., Musaev, F. A., \& Galeev, A. I. 1999, Astron. Lett., 25, 656

Galazutdinov, G. A., Musaev, F. A., Krełowski, J., \& Walker, G. A. H. 2000, PASP, 112, 648

Galazutdinov, G., Petlewski, A., Musaev, F., et al. 2002, A\&A, 395, 969

Grady, C. A., Perez, M. R., \& Thé, P. S. 1994, in The Nature and Evolutionary Status of Herbig Ae/Be Stars, ed. P. S. Thé, M. R. Perez, \& E. P. J. van den Heuvel (San Francisco: ASP), ASP Conf. Ser., 62, 409

Harker, D. E., Wooden, D. H., \& Woodward, C. E. 2002, AAS, 201, 2011

Heger, M. L. 1921, Lick Obs. Bull., 10, 146

Herbig, G. H. 1995, ARA\&A, 33, 19

Herbig, G. H. 1960, ApJS, 4, 337

Hinz, P. M., Hoffmann, W. F., \& Hora, J. L. 2001, ApJ, 561, 131

Hovhannessian, R. Kh., \& Hovhannessian, E. R. 2001, Astrophys., 44, 454

Jaschek, M., Jaschek, C., Andrillat, Y., \& Houziaux, L. 1992, MNRAS, 254, 413

Josafatsson, K., \& Snow, T. P. 1987, ApJ, 319, 436

Krełowski, J., \& Walker, G. A. H. 1987, ApJ, 312, 860

Krełowski, J., \& Westerlund, B. E. 1988, A\&A, 190, 339

Krełowski, J., Galazutdinov, G. A., \& Musaev, F. A. 1998, ApJ, 493, 217

Mannings, V., Koerner, D. V., \& Sargent, A. I. 1997, Nature, 388, 127

Mannings, V., \& Sargent, A. I. 1997, ApJ, 490, 792

Manoj, P., Maheswar, G., \& Bhatt, H. C. 2002, MNRAS, 334, 419

Martin, C., Bouret, J.-C., Deleuil, M., et al. 2002, AAS, 201, 4913

Merrill, P. W. 1950, ApJ, 113, 55

Miroshnichenko, A. S., Mulliss, C. L., Bjorkman, K. S., et al. 1999, MNRAS, 302, 612

Miroshnichenko, A. S., Bjorkman, K. S., Chentsov, E. L., et al. 2001, A\&A, 377, 854

Mora, A., Merin, B., Solano, E., et al. 2001, A\&A, 368, 116

Morrison, N. D., \& Beaver, M. 1995, BAAS, 27, 825 
Moutou, C., Krełowski, J., D’Hendecourt, L., \& Jamroszczak, J. 1999, Sitko, M. L., Grady, C. A., Lynch, D. K., \& Russel, R. W. 1999, ApJ, A\&A, 351, 680 510, 408

Musaev, F. A., Galazutdinov, G. A., Sergeev, A. V., Karpov, N. V., \& Podyachev, Yu. V. 1999, Kinematika Fiz. Nebesn. Tel, 15, No. 3

Natta, A., Grinin, V., \& Mannings, V. 2000, in Protostars and Planets, IV (Tucson: Univ. Arizona Press), 559

Pierce, A. K., \& Breckinridge, J. B. 1973, KPNO preprint, 1063

Pogodin, M. A. 1997, A\&A, 317, 185

Struve, O., \& Swings, P. 1942, ApJ, 96, 475

Surdej, J., \& Swings, P. 1976, A\&A, 47, 113

Surdej, J., \& Swings, P. 1977, A\&A, 54, 219

Thé, P. S., de Winter, D., \& Pérez, M. R. 1994, A\&AS, 104, 315

Vieira, S. L. A., Pogodin, M. A., \& Franco, G. A. P. 1999, A\&A, 345, 559

Rodgers, B., Wooden, D. H., Grinin, V., Shakhovsky, D., \& Natta, A. Vink, J. S., Drew, J. E., Harries, T. J., \& Oudmaijer, R. D. 2002, 2002, ApJ, 564, 405 MNRAS, 337, 356 